\title{
Tobacco and tuberculosis: could we improve tuberculosis outcomes by helping patients to stop smoking?
}

\author{
Jean-Pierre Zellweger ${ }^{1}$, Adithya Cattamanchi ${ }^{2,3}$ and Giovanni Sotgiu ${ }^{4}$
}

Affiliations: ${ }^{1}$ TB Competence Center, Swiss Lung Association, Berne, Switzerland. ${ }^{2}$ Division of Pulmonary and Critical Care Medicine, San Francisco General Hospital, University of California San Francisco, San Francisco, CA, USA. ${ }^{3}$ Curry International Tuberculosis Center, San Francisco General Hospital, University of California San Francisco, San Francisco, CA, USA. ${ }^{4}$ Clinical Epidemiology and Medical Statistics Unit, Department of Biomedical Sciences - University of Sassari - Research, Medical Education and Professional Development Unit, AOU Sassari, Sassari, Italy.

Correspondence: Giovanni Sotgiu, University of Sassari Medical School, Dept of Biomedical Sciences, University of Sassari, via Padre Manzella, 4, Sassari 07100, Italy. E-mail: gsotgiuduniss.it

@ERSpublications

Strengthening smoking control measures may impact on our progress towards tuberculosis elimination http://ow.ly/GmeGL

Tuberculosis disease continues to represent a global threat, with an estimated 9000000 incident cases and 1500000 deaths in 2013 attributable to Mycobacterium tuberculosis infection [1]. Although important progress has been made in the control of the disease from a clinical (i.e. improved clinical outcomes) and public health (i.e. reduced M. tuberculosis transmission in high-, middle- and low-income countries) perspective, the epidemic continues to be fuelled by the persistence of long-lasting triggers.

The major drivers of the epidemic in the last two decades have been the emergence and spread of HIV/ AIDS and multidrug/extensively drug-resistant tuberculosis, with a relevant clinical, political and global economic impact [2-7]. However, several other risk factors have played, and will continue to play, a role [8-12]. One of the most important of these other risk factors, the effect of which on tuberculosis was underestimated for several years, is tobacco smoking, including second-hand smoking.

An important systematic review/meta-analysis, published in 2007, assessed the findings in the scientific literature related to the association between smoking and risk of $M$. tuberculosis infection, tuberculous disease, and tuberculosis-related death [13]. Among six studies, smoking was associated with an approximately two-fold increase in the odds of infection when using either a 5-mm (pooled OR 2.08) or 10-mm (pooled OR 1.83) tuberculin skin test reaction. Among 23 studies, current, former and ever-smokers had an increased odds of tuberculous disease when results were pooled within strata defined by important study characteristics. Finally, all five studies identifying smoking and tuberculosis mortality found a positive association between the two. Five studies explored the risk of tuberculous disease related to passive smoking and found a significantly higher probability of developing tuberculosis in children than in adults (pooled OR 6.52 versus 2.44, respectively).

More recently, two analyses of the population-based 1999-2000 US National Health and Nutrition Examination Survey dataset demonstrated an association between either self-reported smoking history or cotinine-based assessment of smoking status and tuberculous infection.

This issue of the European Respiratory Journal contains two important longitudinal studies that further contribute to our understanding of the impact of tobacco smoking on tuberculosis $[14,15]$.

Received: Dec 012014 | Accepted: Dec 162014

Conflict of interest: None declared.

Copyright OERS 2015 
The first study, by Leung et al. [14], conducted in a large population of tuberculosis patients observed in Hong Kong, China, between 2001 and 2003, and followed for 10 years, confirmed that smokers with tuberculosis had a more severe clinical and radiological presentation, more frequent sputum positivity at presentation and after 2 months of treatment, a lower rate of success and, if successfully cured, a higher risk of relapse. Surprisingly, these findings were observed in spite of the fact that smokers were offered brief advice for smoking cessation, and referral for further nonpharmacological and pharmacological intervention if needed. The study did not, however, assess the rate of cessation among patients with tuberculosis and the impact of cessation on the outcome of tuberculosis or the risk of relapse.

The second study, conducted in Kinshasa, Democratic Republic of Congo, by VANDEN Driessche et al. [15], in a group of patients with tuberculosis and HIV infection, observed that smoking patients were less likely to start antiretroviral therapy during tuberculosis treatment and that the outcome of treatment was less favourable among smokers than nonsmokers, mainly due to a higher rate of loss to follow-up in smokers. The mortality rate during treatment was not different between smokers and nonsmokers, in contrast to the study by LEUNG et al. [14].

These two studies indicate that patients with tuberculosis who are smokers have an increased risk of nearly all aspects of tuberculous disease, namely the severity of clinical presentation, the duration of sputum and culture positivity, unfavourable outcome, and risk of relapse. One of the important issues is that smokers have a prolonged period of contagiousness and may transmit mycobacteria to their contacts for a longer period than nonsmokers, both before the diagnosis and after the initiation of treatment. Furthermore, the fact that smokers with tuberculosis had a higher frequency of loss to follow-up is a worrying signal, as it may imply that some smokers still propagate M. tuberculosis strains in the community without being cured. Therefore, the impact of smoking not only concern smokers with tuberculosis but all of society.

The population-attributable fraction of tuberculosis associated with smoking has been addressed by several studies [16, 17]. A conservative estimate concludes that some $13-20 \%$ of all tuberculosis cases worldwide can be attributed to tobacco smoking, with wide variations between regions, and even higher fractions likely in countries with a high burden of tuberculosis and a high proportion of smokers in the population $[18,19]$. Interventions to decrease the prevalence of smoking among patients with tuberculosis (or in the general population) may have an important impact on the incidence of tuberculosis [20]. Therefore, the World Health Organization (WHO), the International Union Against Tuberculosis and Lung Disease, and the European Respiratory Society insist that the issue of smoking be addressed within the framework of tuberculosis management and care, and that efforts be undertaken to help smoking tuberculosis patients to stop [21].

In this context, two points may surprise: the first is that there are very few published studies on the impact of smoking cessation on the outcome of tuberculosis. One of the only studies was conducted in Sudan and clearly demonstrated that tuberculosis patients cared for by healthcare workers who had been trained in smoking counselling had a better outcome than those who were not [22]. Other studies from different regions, particularly countries with a high prevalence of both tuberculosis and smoking, are urgently needed.

The second point is that interventions including counselling of smoking tuberculosis patients are still far from being implemented in routine clinical practice, particularly in countries where a large proportion of tuberculosis patients are smokers. Considering the negative impact of smoking on the outcome and transmission of tuberculosis, and the potential gain associated with cessation, it is a clear duty of all healthcare workers managing tuberculosis patients (and many of them are respiratory physicians) to address this important issue with their patients.

Smoking continues to be the most important cause of preventable disease and death worldwide, even if awareness of its dangers and measures to limit its impact are taken in many countries, largely as a consequence of the WHO framework convention on tobacco control [23]. Strengthening measures for the control of smoking may have an additional and welcome impact on our progress towards tuberculosis elimination [24-26].

\section{References}

1 World Health Organization. Global Tuberculosis report 2014. Report No. WHO/HTM/TB/2014.08. Geneva, WHO, 2014.

2 Raviglione MC. The TB epidemic from 1992 to 2002. Tuberculosis (Edinb) 2003; 83: 4-14.

3 Glaziou P, Falzon D, Floyd K, et al. Global epidemiology of tuberculosis. Semin Respir Crit Care Med 2013; 34: 3-16.

4 Falzon D, Mirzayev F, Wares F, et al. Multidrug-resistant tuberculosis around the world: what progress has been made? Eur Respir J 2015; 45: 150-160.

5 Tanimura T, Jaramillo E, Weil D, et al. Financial burden for tuberculosis patients in low- and middle-income countries: a systematic review. Eur Respir J 2014; 43: 1763-1775. 
6 Sotgiu G, Mauch V, Migliori GB, et al. Evidence-based, agreed-upon health priorities to remedy the tuberculosis patient's economic disaster. Eur Respir J 2014; 43: 1563-1566.

7 Migliori GB, Sotgiu G, D'Ambrosio L, et al. TB and MDR/XDR-TB in European Union and European Economic Area countries: managed or mismanaged? Eur Respir J 2012; 39: 619-625.

8 Ferrara G, Murray M, Winthrop K, et al. Risk factors associated with pulmonary tuberculosis: smoking, diabetes and anti-TNF $\alpha$ drugs. Curr Opin Pulm Med 2012; 18: 233-240.

9 Zellweger JP. Tobacco and tuberculosis. Monaldi Arch Chest Dis 2008; 69: 83-85.

10 Guidelines on the management of latent tuberculosis infection. World Health Organization, Geneva, 2015. WHO/ HTM/TB/2015.01. Geneva, WHO, 2015.

11 Creswell J, Raviglione M, Ottmani S, et al. Tuberculosis and noncommunicable diseases: neglected links and missed opportunities. Eur Respir J 2011; 37: 1269-1282.

12 Odone A, Houben RM, White RG, et al. The effect of diabetes and undernutrition trends on reaching 2035 global tuberculosis targets. Lancet Diabetes Endocrinol 2014; 2: 754-764.

13 Lin HH, Ezzati M, Murray M. Tobacco smoke, indoor air pollution and tuberculosis: a systematic review and meta-analysis. PLoS Med 2007; 4: e20.

14 Leung CC, Yew WW, Chan CK, et al. Smoking adversely affects treatment response, outcome and relapse in tuberculosis. Eur Respir J 2015; 45: 738-745.

15 Vanden Driessche K, Patel MR, Mbonze N, et al. Effect of smoking history on outcome of patients diagnosed with TB and HIV. Eur Respir J 2015; 45: 839-842.

16 Pai M, Mohan A, Dheda K, et al. Lethal interaction: the colliding epidemics of tobacco and tuberculosis. Expert Rev Anti Infect Ther 2007; 5: 385-391.

17 Slama K, Chiang CY, Enarson DA, et al. Tobacco and tuberculosis: a qualitative systematic review and meta-analysis. Int J Tuberc Lung Dis 2007; 11: 1049-1061.

18 van Zyl-Smit RN, Brunet L, Pai M, et al. The convergence of the global smoking, COPD, tuberculosis, HIV, and respiratory infection epidemics. Infect Dis Clin North Am 2010; 24: 693-703.

19 Basu S, Stuckler D, Bitton A, et al. Projected effects of tobacco smoking on worldwide tuberculosis control: mathematical modelling analysis. BMJ 2011; 343: d5506.

20 Lin HH, Murray M, Cohen T, et al. Effects of smoking and solid-fuel use on COPD, lung cancer, and tuberculosis in China: a time-based, multiple risk factor, modelling study. Lancet 2008; 372: 1473-1483.

21 World Health Organization. A WHO/The Union monograph on TB and tobacco control: joining efforts to control two related global epidemics. Geneva, WHO, 2007.

22 El Sony A, Slama K, Salieh M, et al. Feasibility of brief tobacco cessation advice for tuberculosis patients: a study from Sudan. Int J Tuberc Lung Dis 2007; 11: 150-155.

23 World Health Organization. WHO report on the global tobacco epidemic 2013. Geneva, WHO, 2013.

24 Diel R, Loddenkemper R, Zellweger JP, et al. Old ideas to innovate tuberculosis control: preventive treatment to achieve elimination. Eur Respir J 2013; 42: 785-801.

25 D'Ambrosio L, Dara M, Tadolini M, et al. Tuberculosis elimination: theory and practice in Europe. Eur Respir J 2014; 43: 1410-1420.

26 Dye C, Glaziou P, Floyd K, et al. Prospects for tuberculosis elimination. Annu Rev Public Health 2013; 34 $271-286$. 